CIENCIA Y SOCIEDAD

Volumen XXIX, Número 1

Enero-Marzo 2004

\title{
UNA ALTERNATIVA PARA PRODUCCIÓN DE FRÍO CON ENERGÍA SOLAR
}

\section{Inna Samson* \\ Rodolfo Echarri**}

\section{RESUMEN}

En el presente trabajo se hace referencia a las distintas técnicas de refrigeración que se conocen en la actualidad con una breve descripción de cada una de ellas para finalizar con la elección que parece ser la más conveniente para la República Dominicana.

Además se realiza una primera aproximación al dimensionamiento de un refrigerador que utiliza la radiación solar como fuente de energía y el fenómeno de adsorción de metanol en carbón activado para la producción del frío. Para dicho cálculo, se tienen en cuenta las condiciones ambientales de República Dominicana.

PALABRAS CLAVES

Refrigeracion, nevera, radiación solar.

* Departamento de Ciencias Básicas, Instituto Tecnológico Santo Domingo. Republica Dominicana.

** Instituto del Desarrollo Humano, Universidad Nacional de General Sarmiento, República Argentina 


\section{Introducción}

Los orígenes del tipo de refrigeración que planteamos en el presente trabajo, se remontan a finales del siglo XVIII', pero si tenemos en cuenta el hecho de que en la antigüedad a veces se llevaba nieve compactada desde las montañas hasta los palacios reales, podríamos pensar que el interés por la obtención de frío tiene un origen mucho anterior.

\section{Elección del sistema a desarrollar}

Para elegir el sistema óptimo para las condiciones reinantes en República Dominicana, hemos hecho un sondeo de los diferentes tipos de refrigeración que existen en el presente, evaluando tanto el costo de producción, como las posibilidades de su realización con la tecnología a la que se puede acceder en el país en forma inmediata sin elevados costos de importación. Por otro lado se piensa que la formación de un grupo de investigadores en este rubro nos dará, en el futuro, la posibilidad de mejorar el estado tecnológico actual para la producción de neveras más sofisticadas una vez que la propuesta en el presente trabajo se encuentre en la etapa de producción.

A continuación presentamos una pequeña reseña de las posibles formas de lograr producción de frío, con el propósito de brindar al lector una visión tanto de las distintas posibilidades como del "estado del arte" así como las limitaciones de cada una de ellas. 


\section{Refrigeración por compresión}

Este sistema, es el utilizado en las neveras hogareñas, y en la mayoría de las instalaciones industriales de producción de frío. El funcionamiento del mismo se basa en comprimir un gas hasta licuarlo, eliminando el calor que se produce al ambiente por medio de un radiador. Luego de esto, el líquido obtenido se evapora en una cámara térmicamente aislada del ambiente, con lo que se obtiene el frío en dicha cámara.

Los gases más usados para este tipo de equipos son los clorofluorcarbonos conocidos como CFC, y que tienen consecuencias graves en la disminución de la capa de ozono. En algunas instalaciones industriales se utiliza el amoníaco, que si bien no tiene consecuencias sobre la capa de ozono, tiene consecuencias directas sobre la salud, con lo que la explotación de este tipo de tecnología requiere de controles estrictos.

La utilización de este tipo de dispositivos con energía solar nos llevaría a la importación de células fotoeléctricas, con el consiguiente costo de importación.

\section{Efecto Peltier}

Este efecto fue descubierto por J. Peltier a principios del siglo XIX y consiste en que el paso de una corriente eléctrica a través de la unión de dos metales distintos, es capaz de enfriar la juntura si circula en el sentido correcto. Este efecto suele ser enmascarado por el efecto Joule, que calienta la juntura. 
Sin embargo, eligiendo en forma correcta el par de metales utilizados, como también el dimensionamiento del dispositivo, es posible lograr frío en forma directa a partir del pasaje de una corriente.

Actualmente existen refrigeradores de este tipo (llamados refrigeradores termoeléctricos) en los que se utiliza no ya una juntura de dos metales, sino elementos de estado sólido. Dichos refrigeradores presentan un rendimiento mucho menor que los refrigeradores de compresión estandar (los de uso doméstico). Sin embargo, cuando se trata de producir frío en un pequeño volumen ( por ejemplo en dispositivos electrónicos como una CCD de bajo ruido) el rendimiento de los refrigeradores a compresor cae drásticamente y resulta mucho más conveniente el uso de los termoeléctricos. Incluso existen pequeñas unidades que permiten mantener frías las bebidas y comestibles para un día de camping conectando la unidad al encendedor del carro. Uno de los inconvenientes mayores de estas unidades es que cuando se interrumpe el paso de la corriente eléctrica, la zona del refrigerador que era muy eficiente para hacer pasar el calor desde el interior del refrigerador hacia el medio ambiente se convierte en una zona de grandes pérdidas del frío logrado. De este modo, frente a una interrupción del suministro de energía eléctrica, la temperatura del interior sube rápidamente hasta llegar a la del ambiente.

Las principales ventajas se encuentran en su pequeño tamaño, son silenciosas porque no poseen partes móviles, y su 
relativamente larga vida útil. Además no contienen líquidos ni gases como medios refrigerantes, lo que las hace inocuas para el medio ambiente ( $s i$ no se piensa en que es necesario producir la energía eléctrica con la que se las debe alimentar).

Por otro lado, la utilización de estos dispositivos haciendo uso de la energía solar, implica la necesidad de importación de celdas fotovoltaicas, así como los componentes de estado sólido que extraen el calor.

En este caso, no tenemos la posibilidad de competir con las grandes potencias productoras de este tipo de dispositivos, ya que nos llevan muchos años de ventaja, y poseen enormes grupos de desarrollo tecnológico dedicados a este tipo de tecnología.

\section{Refrigeración termoacústica}

Los dispositivos de este tipo ${ }^{2}$ son básicamente, un tubo metálico hueco cerrado en un extremo. En el otro extremo se encuentra un diafragma vibrante que produce la onda sonora sobre el gas encerrado en el tubo. Las fluctuaciones de presión en la cavidad son acompañadas por fluctuaciones de temperatura, y de esta forma, el calor es transportado desde el extremo cerrado del tubo hacia las cercanías del diafragma, donde se encuentra una pieza de material poroso que recibe el calor y lo expulsa al medio ambiente.

Este tipo de dispositivo se encuentra aún en una etapa ex- 
perimental, considerándose por ejemplo la posibilidad de refrigerar chips de computadoras. Por lo tanto, esta tecnología no tiene en el presente posibilidades reales de aplicación para refrigeradores hogareños.

\section{Refrigeración por absorción}

El sistema de refrigeración por absorción consiste en ciclos donde una sustancia es disuelta en otra y la cantidad de sustancia disuelta cambia por la aplicación de calor a la mezcla. Luego de esto, la sustancia que se evaporó de la mezcla es reabsorbida por la misma, momento en que se produce la refrigeración.

El primer sistema fue desarrollado por John Leslie utilizando el par ácido sulfúrico-agua, donde el ácido sulfúrico reabsorbía el agua y esta, por evaporación, enfriaba un recipiente. Más tarde, en 1859, Ferdinand Carré desarrolla una máquina refrigeradora que utilizaba el par amoníaco - agua, donde el enfriado se produce por la evaporación del amoníaco.

Actualmente los pares más usados para la refrigeración por absorción son el amoníaco-agua y agua-bromuro de litio.

El primero de ellos tiene la ventaja de que se puede usar por debajo del punto de congelación del agua porque el refrigerante es el amoníaco, mientras que para el segundo par, el refrigerante es el agua, con lo que es difícil conseguir temperaturas cercanas al $0^{\circ} \mathrm{C}$. 
Durante los primeros años del siglo XX, Leo Szilard patentó un ciclo refrigerante que utiliza el par amoníaco agua más un gas adicional, el hidrógeno, para producir la evaporación del amoníaco por caída de su presión parcial, mientras que Einstein patentó a su vez, un ciclo de amoníaco agua, butano. Mientras que en el ciclo de Szilard, el refrigerante es el amoníaco, en el de Einstein, el refrigerante es el butano. Recién en la década del ' 90 se realizó la comprobación experimental del funcionamiento del ciclo de Einstein ${ }^{3}$, mientras que las neveras de Szilard han funcionado desde hace muchos años con pleno éxito, siendo éstas las que producen el frío quemando gas butano o queroseno en las zonas rurales donde no existe la energía eléctrica. Este tipo de tecnología se encuentra bien desarrollada pero necesita una fuente caliente de alta temperatura para que su rendimiento sea aceptable. Esto llevaría a utilizar concentradores solares si se pretende utilizar al sol como fuente de energía, lo que lleva a altos costos de producción y mantenimiento de los equipos para el amoníaco.

En el caso del bromuro de litio, la fuente caliente no necesita temperaturas tan elevadas como para utilizar concentradores, pero el sistema se encarece por el precio del bromuro.

\section{Refrigeración por adsorción}

La refrigeración de este tipo, se basa en los procesos de asdorción y desorción ${ }^{4,5}$ de una materia en estado gaseoso sobre un cuerpo sólido. 
En el proceso de adsorción, el gas es atrapado por la superficie del cuerpo sólido liberando una cierta cantidad de energía en forma de calor. El proceso de desorción es, por el contrario, la liberación del gas atrapado por el sólido, cuando a éste se le entrega calor.

Si bien estos fenómenos se aplican desde hace muchísimo tiempo en la técnica de procesos industriales, en el campo de la refrigeración se aplicaron en los años veinte y treinta del siglo XX pero fueron desplazadas rápidamente por la aplicación de los CFC a las máquinas que funcionan con ciclo de compresión.

Una de las razones más notables para que esto ocurriera, es el carácter intermitente de su funcionamiento, ya que se produce frío sólo en la etapa de adsorción. Sin embargo, a partir de la década del '70, con la crisis energética mundial sumada a los problemas ambientales suscitados por los CFC, comienza a despertarse un nuevo interés en el desarrollo de este tipo de refrigeración.

\section{Sistema elegido}

Nuestra elección del sistema de refrigeración se ha basado en dos aspectos fundamentales; la facilidad de construcción de un prototipo con las posibilidades actuales en República Dominicana, y la relación costo rendimiento de los diferentes ciclos haciendo uso de la energía solar ${ }^{6.7}$ así por ejemplo 
se podría hacer uso del efecto Peltier y células fotovoltaicas, pero esto produciría un costo dependiente de la importación de estos elementos.

También se pensó en un principio en hacer uso de un ciclo de Szilard o Einstein, pero estos necesitan una alta temperatura de la fuente caliente, lo que implica el uso no ya de colectores solares sino de concentradores, con la consiguiente complicación y elevado costo del sistema resultante.

Para las posibilidades de trabajo con las que se cuenta, tanto en la producción del dispositivo como las condiciones de operación del mismo, uno de los ciclos que parece ser el más conveniente es el de adsorción a pesar de la naturaleza intermitente de su funcionamiento. El par refrigerante elegido es el metanol - carbón activado o el etanol - carbón activado.

Si tenemos en cuenta que una de las materias primas preferidas en la producción del carbón activado es la cáscara del coco a la que se calcina en una atmósfera controlada, y que el etanol se produce por fermentación y destilación de la caña de azúcar, el segundo de los pares mencionados parece ser el más conveniente. Sin embargo, preferimos construir un primer prototipo utilizando metanol ya que ese sistema ya ha sido probado en otros lugares con resultados prometedores. Una vez puesto en funcionamiento y evaluado dicho prototipo, se cambiará el metanol por etanol para realizar la comparación entre ambos y decidir la conveniencia de uno u otro. 


\section{Principio de funcionamiento:}

El equipo propuesto ${ }^{5.89}$ consta de un colector - generador, un condensador, un evaporador y la cámara fría como se puede observar en la figura 1.
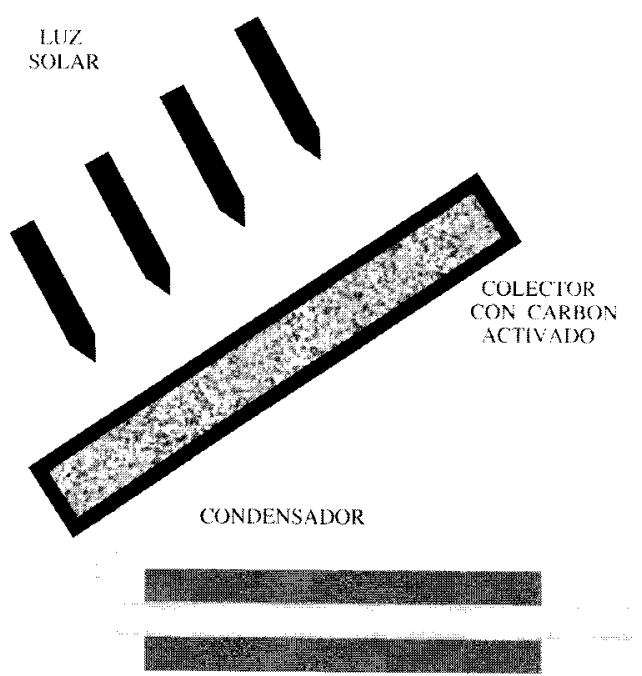

FVAPORADOR

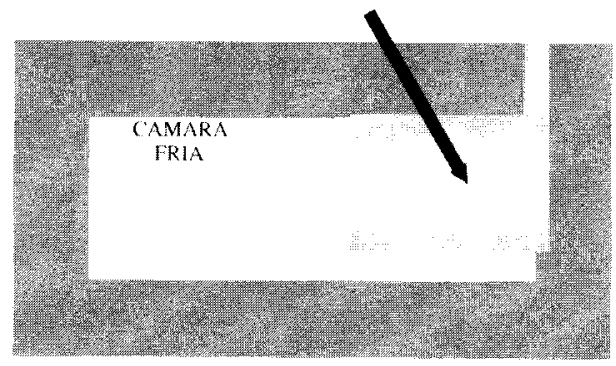


El principio de funcionamiento es el siguiente; al comenzar la jornada, el colector y el carbón activado que contiene el mismo, se encuentran a una temperatura que es la ambiente $\mathrm{T}_{\mathrm{a}}$ entonces todo el metanol del que dispone el sistema se encuentra adsorbido en el carbón activado. A medida que avanza el día, el colector va recibiendo la radiación solar y elevando su temperatura (junto con la del carbón activado) de forma que el metanol se va desorbiendo y pasando al condensador al tiempo que su presión va aumentando hasta que alcanza la presión de condensación $\mathrm{P}_{\mathrm{c}}$ a la temperatura del condensador $\mathrm{T}_{c}$. Apartir de ese momento, la presión de vapor de metanol en el sistema permanece constante mientras que siga existiendo desorción. De esta forma, se obtiene metanol en forma líquida y el calor de condensación es eliminado hacia el ambiente desde el condensador.

Una vez avanzado el día, la radiación solar comienza a decrecer con el consiguiente descenso de la temperatura del colector. De esta forma, el metanol comienza a ser readsorbido lentamente por el carbón activado si en ese momento se abre la válvula que conecta el condensador con el evaporador, el metanol líquido pasa al evaporador por gravedad y su evaporación se produce dentro del mismo. De esta forma, elcalor necesario para la evaporación del metanol es tomado de la cámara fría, donde el fenómeno es utilizado por ejemplo para enfriar agua y producir hielo. 


\section{Dimensionamiento}

Lo primero que definiremos para realizar el dimensionamiento de nuestro sistema, es la cantidad de hielo que queremos producir.

Supondremos para fijar ideas que queremos obtener $5 \mathrm{~kg}$ de hielo por jornada partiendo de agua a $25^{\circ} \mathrm{C}$. Para esto, el evaporador deberá extraer el calor suficiente de la cámara fría para bajar la temperatura del agua de $25^{\circ} \mathrm{C}$ a $0^{\circ} \mathrm{C}$ y luego solidificarla.

La capacidad calorífica del agua es aproximadamente $1 \mathrm{cal} / \mathrm{g}=4.18 \mathrm{~J} / \mathrm{g}$. De acuerdo a esto, el calor que es necesario extraer para llevarla a la temperatura de congelación será:

$$
Q_{1}=C m \Delta t
$$

Donde $\boldsymbol{C}$ es la capacidad calorífica $\boldsymbol{m}$ la masa de agua y $\Delta t$ la diferencia de temperaturas.

En nuestro caso :

$$
Q_{1}=4,18\left(\mathrm{~J} / \mathrm{g}^{\circ} \mathrm{C}\right) 500 \mathrm{~g} 25^{\circ} \mathrm{C}=522500 \mathrm{~J}
$$

Una vez lograda la temperatura de congelación hace falta extraer el calor de solidificación $\mathbf{Q}_{2}$ para lograr hielo está relacionado con la masa del mismo y con el calor latente de fusión $\mathbf{L}=333.15 \mathbf{J} / \mathbf{g}$. 


$$
Q_{2}=L \boldsymbol{m}
$$

Que con nuestros datos se convierte en:

$$
Q_{2}=333.15(\mathrm{~J} / \mathrm{g}) 500 \mathrm{~g}=1665750 \mathrm{~J}
$$

El calor que es necesario extraer de la cámara fría para producir $5 \mathrm{Kg}$ de hielo será:

$$
Q=Q_{1}+Q 2=2188250 J
$$

Suponiendo que la cámara fría es perfectamente adiabática, dicho calor es exactamente igual al que tiene que extraerse por medio de la evaporación del metanol. Dado que el calor de evaporación del metanol es conocido $\left(\mathbf{L}_{\mathrm{m}}=1133 \mathrm{~J} / \mathrm{g}\right)$ podemos calcular la cantidad a evaporar.

$$
m_{m}=Q / L_{m}=\frac{2188250 \mathrm{~J}}{1133(\mathrm{~J} / \mathrm{g})}=1931 \mathrm{~g}=2 \mathrm{Kg}
$$

Este valor, nos permite calcular la cantidad de carbón activado que es necesario colocar en el colector de la siguiente forma:

La temperatura máxima que alcanza un colector solar plano típico, es de unos $100^{\circ} \mathrm{C}$ y la mínima es la ambiente nocturna que en República Dominicana tiene una media de $25^{\circ} \mathrm{C}$. De esta forma $\mathbf{m}_{\mathbf{m}}$ es la diferencia de las masas de metanol adsorbidas a $25^{\circ} \mathrm{C}$ y a $100^{\circ} \mathrm{C}$ por el carbón activado. 
La ecuación de Dubinin - Radushkevich para un carbón activado comercial típico ${ }^{10}$ es

$$
X=e^{-1.1210^{-6} o K^{-2}\left(T \ln \left(P_{s} / P\right)^{2}\right.}
$$

donde:

$\mathrm{X}$ es la concentración: masa de adsorbato (metanol)/masa de adsorbente(carbón activado)

T la temperatura absoluta del carbón activado

Ps Presión del vapor saturado del adsorbato a la temperatura $\mathrm{T}$

P Presión de equilibrio a la misma temperatura $T$

La presión de vapor del metanol en función de la temperatura se da en la tabla 1.

\begin{tabular}{|c|c|c|c|c|c|}
\hline $\begin{array}{c}\text { Temperatura } \\
{ }^{\circ} \mathrm{C}\end{array}$ & $\begin{array}{c}\text { Presión de } \\
\text { vapor } \mathrm{mmHg}\end{array}$ & $\begin{array}{c}\text { Temperatura } \\
{ }^{\circ} \mathrm{C}\end{array}$ & $\begin{array}{c}\text { Presión de } \\
\text { vapor } \mathrm{mmHg}\end{array}$ & $\begin{array}{c}\text { Temperatura } \\
{ }^{\circ} \mathrm{C}\end{array}$ & $\begin{array}{c}\text { Presión de } \\
\text { vapor } \mathrm{mmHg}\end{array}$ \\
\hline-10 & 15.61 & 30 & 163.97 & 70 & 938.26 \\
\hline-5 & 21.88 & 35 & 209.67 & 75 & 1131.03 \\
\hline 0 & 30.24 & 40 & 265.75 & 80 & 1355.47 \\
\hline 5 & 41.26 & 45 & 334.04 & 85 & 1615.40 \\
\hline 10 & 55.59 & 50 & 416.51 & 90 & 1914.96 \\
\hline 15 & 74.02 & 55 & 515.64 & 95 & 2258.56 \\
\hline 20 & 97.48 & 60 & 633.72 & 100 & 2650.91 \\
\hline 25 & 127.05 & 65 & 773.59 & $\ldots$ & \\
\hline
\end{tabular}

Tabla 1. Presión de vapor de metanol en función de la temperatura

La presión del metanol en el colector durante la desorción es la de saturación en el condensador a la temperatura de condensación (que podemos suponer de unos $30^{\circ} \mathrm{C}$ ), mientras que durante el ciclo de adsorción es la de evaporación de la cámara fría (que suponemos de $0{ }^{\circ} \mathrm{C}$ ) 
Con estos datos, podemos realizar la tabla siguiente (tabla 2):

\begin{tabular}{|c|c|c|c|c|c|}
\hline & Ps mmHg & P mmHg & T condensador ${ }^{\circ} \mathrm{C}$ & T colector ${ }^{\circ} \mathrm{C}$ & $X$ \\
\hline Desorción & $265 \mathrm{l}$ & 164 & 30 & 100 & 0,30 \\
\hline Adsorción & 164 & 30 & 25 & 30 & 0,74 \\
\hline
\end{tabular}

Tabla 2

De lo anterior, vemos que la diferencia de metanol contenido en el carbón activado es de $0,44 \mathrm{Kg}$ de metanol por kilogramo de carbón activado. Por otro lado, dado que el calor latente de evaporación para el metanol es de $1204 \mathrm{~J} / \mathrm{g}$, y que se deben extraer 2188250 joules, la masa de metanol necesaria para hacerlo será de :

$$
m_{m d}=\frac{2188250 \mathrm{~J}}{1204(\mathrm{~J} / \mathrm{g})}=1817,5 \mathrm{~g}
$$

De aquí es inmediato el cálculo de la cantidad de carbón activado necesaria para obtener esa cantidad de metanol en el evaporador.

$$
m_{c}=\frac{m_{m d}}{044}=\frac{1,8 \mathrm{Kg}}{0,44}=4,13 \mathrm{Kg}
$$

Además, hay que tener en cuenta que en el momento en que la masa de metanol calculada se encuentra en el evaporador, en el carbón activado todavía queda un remanente de metanol que es de 


$$
Q_{m r}=m_{c} 0,03=1,24 \mathrm{Kg}
$$

Entonces la masa total de metanol en el sistema será

$$
m_{m}=m_{r n d}+m_{m}=2,06 \mathrm{Kg}
$$

Por otro lado, teniendo en cuenta el peso específico del carbón activado $(0,5 \mathrm{Kg} / \mathrm{dm} 3)$ y del metanol $\left(0,8 \mathrm{Kg} / \mathrm{dm}^{3)}\right.$ podemos ver que el volumen de los dos elementos en juego es del orden de los diez litros, lo que nos da una idea del tamaño del equipo aunque el carbón activado se encuentre formando una delgada capa dentro del colector solar.

En el caso del etanol, la tabla 3 da las presiones de vapor en función de la temperatura

\begin{tabular}{|c|c|c|c|c|c|}
\hline $\begin{array}{c}\text { Temperatura } \\
{ }^{\circ} \mathrm{C}\end{array}$ & $\begin{array}{c}\text { Presión de } \\
\text { vapor } \mathrm{mmHg}\end{array}$ & $\begin{array}{c}\text { Temperatura } \\
{ }^{\circ} \mathrm{C}\end{array}$ & $\begin{array}{c}\text { Presión de } \\
\text { vapor mmHg }\end{array}$ & $\begin{array}{c}\text { Temperatura } \\
{ }^{\circ} \mathrm{C}\end{array}$ & $\begin{array}{c}\text { Presión de } \\
\text { vapor mmHg }\end{array}$ \\
\hline-10 & 5.56 & 30 & 78.47 & 70 & 542.20 \\
\hline-5 & 8.16 & 35 & 103.20 & 75 & 665.95 \\
\hline 0 & 11.77 & 40 & 134.33 & 80 & 812.48 \\
\hline 5 & 16.71 & 45 & 173.17 & 85 & 984.95 \\
\hline 10 & 23.37 & 50 & 2211.19 & 90 & 1186.89 \\
\hline 15 & 32.23 & 55 & 280.9 & 95 & 1421.74 \\
\hline 20 & 43.89 & 60 & 351.77 & 100 & 1693.80 \\
\hline 25 & 59.02 & 65 & 438.35 & -- & \\
\hline
\end{tabular}

Tabla 3. Presión de vapor de etanol en función de la temperatura

Con estos datos, y haciendo uso del hecho que para componentes con comportamientos químicos parecidos la ecuación 22 
de Dubinin - Radushkevich es aproximadamente válida sin ningún cambio en sus coeficientes, podemos realizar la tabla siguiente (tabla4):

\begin{tabular}{|c|c|c|c|c|c|}
\hline & Ps mmHg & $\mathrm{P} \mathrm{mmHg}$ & T condensador ${ }^{\circ} \mathrm{C}$ & T colector ${ }^{\circ} \mathrm{C}$ & $\mathrm{X}$ \\
\hline Desorción & 1694 & 78 & 30 & 100 & 0,3 \\
\hline Adsorción & 78 & 12 & 25 & 30 & 0.7 \\
\hline \multicolumn{7}{|c|}{ Tabla4 }
\end{tabular}

Mientras que con el metanol teníamos una diferencia de masa de $0,44 \mathrm{Kg}$, en el caso del etanol se podrán obtener 0,4 $\mathrm{Kg}$ del mismo por $\mathrm{Kg}$ de carbón activado (apenas una diferencia del 10\%).

Por otro lado, dado que el calor latente del etanol es de 8.54 $10^{2} \mathrm{~J} / \mathrm{g}$

$$
\mathrm{m}_{\mathrm{md}}=\frac{2188250 \mathrm{~J}}{854 \mathrm{~J} / \mathrm{g}}=1817,5 \mathrm{~g}
$$

además

$$
\begin{gathered}
m_{m d}=\frac{2,562 \mathrm{Kg}}{04 \mathrm{~J} / \mathrm{g}}=6,406 \mathrm{Kg} \\
m_{m}=6,406 \mathrm{Kg} 0,3 \\
y \quad m_{m}=m_{m}+m_{m}=4,483 \mathrm{Kg}
\end{gathered}
$$




\section{Conclusiones}

A partir de los cálculos realizados, que por supuesto son una primera aproximación al dimensionamiento de un prototipo, podemos concluir que las masas de metanol y carbón activado (o etanol carbón activado para el segundo caso)puestas en juego son aceptables en relación con la producción de hielo esperada, ya que el tamaño del equipo guarda una relación razonable con la cantidad de hielo a producir que se ha propuesto.

Por otro lado, estamos en condiciones de afirmar que es posible poner en funcionamiento una máquina de este tipo en la República Dominicana, ya que no se necesita de un laboratorio sofisticado para producirla. Sin embargo, para fabricarla, hará falta afinar los cálculos para el correcto diseño del colector solar donde estará contenido el carbón activado, teniendo en cuenta la insolación y temperaturas máximas y mínima promedio para el país, así como el óptimo diseño del disipador a incorporar en el condensador, aislación térmica de la cámara fría, etc.

\section{Bibliografía}

1-. Tania Carbonell Morales. La refrigeración alternativa. Energía y Tu (CUBASOLAR) No19 Julio - Setiembre de 2002

2-. Jay A. Adeff and Thomas J. Hofler. Design and construction of a solar powered, thermoacoustically driven, thermoacoustic refrigerator. Departament of Physics, Naval Postgraduate School. Monterrey, CA 93943, USA 
3-. A. Delano. Analysis of the Einstein refrigeration cycle. Georgia Institute of Technology May 1997.

4-. K. Sumathy. An efficient solar ice maker. Departament of Mechanical Energy, University of Honk Kong

5-. F. Lemini, A. Errougani, F. Bentayeb. Realisation experimentale d'un refrigerateur solaire a adsorption à Rabat. FIER' 2002 Tetouan Maroc

6-. M. Ahachad, A. Almers and E. Mimet. Etude comparatif des machines frigorifiques solaires a adsorption et a absortion. FIER' 2002 Tetouan Maroc.

7-. $J$ hougard. Analyse energetique et entropique de plusieurs cycles de refrigeration solaire a adsorption. FIER' 2002 Tetouan Maroc.

8-. E.E. Anyanwu. Review of solid adsorption solar refrigerator I: an overview of the refrigeration cycle. Energy conversion and Management 44 (2003) $301-312$

9-. E.E. Anyanwu, C.I. Ezekwe. Design, construction and test run of a solid adsorption solar refrigerator using activated carbon/ methanol, as adsorbent/adsorbate pair. Energy Conversion and Management 44 (2003) 2879 - 2892

10-. Erico Frigerio. Sistema solar de refrigeración por adsorción. Características del carbón activado. Universidad Nacional de Salta 